\title{
Lowest order constrained variational calculation of polarized neutron matter at finite temperature
}

\author{
G.H. Bordbar ${ }^{1,3} *$ and M. Bigdeli ${ }^{2,3 \dagger}$ \\ ${ }^{1}$ Department of Physics, \\ Shiraz University, Shiraz 71454, Iran ${ }^{\ddagger}$, \\ ${ }^{2}$ Department of Physics, \\ Zanjan University, \\ P.O. Box 45195-313, Zanjan, Iran \\ and \\ ${ }^{3}$ Research Institute for Astronomy and Astrophysics of Maragha, \\ P.O. Box 55134-441, Maragha, Iran
}

\begin{abstract}
Some properties of the polarized neutron matter at finite temperature has been studied using the lowest order constrained variational (LOCV) method with the $A V_{18}$ potential. Our results indicate that spontaneous transition to the ferromagnetic phase does not occur. Effective mass, free energy, magnetic susceptibility, entropy and the equation of state of the polarized neutron matter at finite temperature are also calculated. A comparison is also made between our results and those of other many-body techniques.
\end{abstract}

\footnotetext{
${ }^{*}$ Corresponding author. E-mail: bordbar@physics.susc.ac.ir

$\dagger$ E-mail: m_bigdeli@znu.ac.ir

$\ddagger$ Permanent address
} 


\section{INTRODUCTION}

The spontaneous phase transition to a ferromagnetic state in the neutron matter is of particular interest in astrophysics. Specially, this transition could have important consequences for the physical origin of magnetic field of pulsars, that are believed to be rapidly rotating neutron stars with strong surface magnetic fields in the range of $10^{12}-10^{13}$ Gauss [1, 2, 3, 4], and also the evolution of a protoneutron star. A hot neutron star is born within a short time after supernovae explosion. In this stage (protoneutron star), the interior temperature of a neutron star is of the order $20-50 \mathrm{MeV}$ [5]. Therefore, the study of magnetic properties of polarized neutron matter at finite temperature is of special interest in the description of protoneutron stars.

There exist several possibilities of the generation of the magnetic field in a neutron star. From the nuclear physics point of view, one is the possible existence of a phase transition to a ferromagnetic state at densities corresponding to the theoretically stable neutron stars and, therefore, of a ferromagnetic core in the liquid interior of such compact objects. Such a possibility has been studied by several authors using different theoretical approaches [6-29], but the results are still contradictory. In most calculations, neutron star matter is approximated by pure neutron matter, as proposed just after the discovery of pulsars. Whereas some calculations, like those of based on hard sphere gas model [6, 7], Skyrmelike interactions [23], Reid soft-core potential [13] and relativistic Dirac-Hartree-Fock approximation with an effective nucleon-meson Lagrangian [18] showed that neutron matter becomes ferromagnetic at some densities. Others, like recent Monte Carlo [22] and Brueckner-Hartree-Fock calculations [21-23] using modern two-body and three-body realistic interactions show no indication of ferromagnetic transition at any density for neutron matter and asymmetrical nuclear matter.

Most of the studies of the ferromagnetic transition in neutron matter and nuclear matter have been done at zero temperature. The properties of polarized neutron matter both at zero and finite temperature have been studied by several authors [29, 30, 31]. Bombaci et al. (BPRRV) [31] have studied the properties of polarized neutron matter within the framework of the Brueckner-Hartree-Fock formalism using the $A V_{18}$ nucleon-nucleon interaction. Their results show no indication of a ferromagnetic transition at any density and temperature. Lopez-Val et al. [30] have used the D1 and D1P parameterization of the Gogny interac- 
tion and the results of their calculation show two different behaviors: whereas the D1P force exhibits a ferromagnetic transition at density of $\rho \sim 1.31 \mathrm{fm}^{-3}$ whose onset increases with temperature, no sign of such a transition is found for D1 at any density and temperature. Rios and Polls [29] have used Skyrme-like interactions and their results indicate the occurrence of a ferromagnetic phase of neutron matter.

Recently, we have computed the properties of polarized neutron matter [32], polarized symmetrical nuclear matter [33], such as total energy, magnetic susceptibility, pressure, etc at zero temperature using the microscopic calculations employing the lowest order constrained variational (LOCV) method with the $A V_{18}$ potential. We have also calculated the properties of spin polarized asymmetrical nuclear matter and neutron star matter [34] using the LOCV method employing the $A V_{18}$ [35], Reid93 [36], $U V_{14}$ [37] and $A V_{14}$ [38] potentials. We have concluded that the spontaneous phase transition to a ferromagnetic state in the neutron matter, symmetrical and asymmetrical nuclear matter and neutron star matter does not occur.

In the present work, we study the properties of polarized neutron matter at finite temperature using the LOCV technique employing the $A V_{18}$ potential.

\section{LOCV FORMALISM FOR POLARIZED HOT NEUTRON MATTER}

We consider a system of $N$ interacting neutrons with $N_{1}$ spin up and $N_{2}$ spin down neutrons. The total number density $(\rho)$ and spin asymmetry parameter $(\delta)$ are defined as

$$
\begin{aligned}
& \rho=\rho_{1}+\rho_{2}, \\
& \delta=\frac{N_{1}-N_{2}}{N} .
\end{aligned}
$$

$\delta$ shows the spin ordering of matter which can have a value in the range of $\delta=0.0$ (unpolarized matter) to $\delta=1.0$ (fully polarized matter).

Now, we consider a trial many-body wave function of the form

$$
\psi=F \phi
$$

where $\phi$ is the uncorrelated ground state wave function (simply the Slater determinant of plane waves) of $N$ independent neutrons and $F=F(1 \cdots N)$ is an appropriate N-body 
correlation operator which can be replaced by a Jastrow form i.e.,

$$
F=\mathrm{S} \prod_{i>j} f(i j)
$$

in which $\mathrm{S}$ is a symmetrizing operator. We consider a cluster expansion of the energy functional up to the two-body term,

$$
E([f])=\frac{1}{N} \frac{\langle\psi \mid H \psi\rangle}{\langle\psi \mid \psi\rangle}=E_{1}+E_{2} .
$$

For hot neutron matter, the one-body term $E_{1}$ is

$$
E_{1}=\sum_{i=1,2} \varepsilon_{i}
$$

Labels 1 and 2 are used instead of spin up and spin down neutrons, respectively, and $\varepsilon_{i}$ is

$$
\varepsilon_{i}=\sum_{k} \frac{\hbar^{2} k^{2}}{2 m} n_{i}\left(k, T, \rho_{i}\right),
$$

where $n_{i}\left(k, T, \rho_{i}\right)$ is the Fermi-Dirac distribution function,

$$
n_{i}\left(k, T, \rho_{i}\right)=\frac{1}{e^{\beta\left[\epsilon_{i}\left(k, T, \rho_{i}\right)-\mu_{i}\left(T, \rho_{i}\right)\right]}+1} .
$$

In the above equation $\beta=\frac{1}{T}$ and $\mu_{i}$ being the chemical potential, $\rho_{i}$ is the number density and $\epsilon_{i}$ is the single particle energy of a neutron with spin projection $i$.

In our formalism, the single particle energy, $\epsilon_{i}$, of a neutron with momentum $k$ and spin projection $i$ is approximately written in terms of effective mass as [29, 30]

$$
\epsilon_{i}\left(k, T, \rho_{i}\right)=\frac{\hbar^{2} k^{2}}{2 m_{i}^{*}(\rho, T)}+U_{i}\left(T, \rho_{i}\right) .
$$

In fact, we use a quadratic approximation for single particle potential, incorporated in the single particle energy as a momentum independent effective mass. $U_{i}\left(T, \rho_{i}\right)$ is the momentum independent single particle potential. The effective mass, $m_{i}^{*}$, is determined variationally [39, 40, 41, 42, 43].

The chemical potentials, $\mu_{i}$, at any adopted values of the temperature $(T)$, number density $\left(\rho_{i}\right)$ and spin polarization $(\delta)$, are determined by applying the constraint,

$$
\sum_{k} n_{i}\left(k, T, \rho_{i}\right)=N_{i}
$$

This is an implicit equation which can be solved numerically. 
The two-body energy $E_{2}$ is

$$
E_{2}=\frac{1}{2 A} \sum_{i j}\langle i j|\nu(12)| i j-j i\rangle,
$$

where

$\nu(12)=-\frac{\hbar^{2}}{2 m}\left[f(12),\left[\nabla_{12}^{2}, f(12)\right]\right]+f(12) V(12) f(12), f(12)$ and $V(12)$ are the two-body correlation and potential. For the two-body correlation function, $f(12)$, we consider the following form [44, 45]:

$$
f(12)=\sum_{k=1}^{3} f^{(k)}(12) O^{(k)}(12)
$$

where, the operators $O^{(k)}(12)$ are given by

$$
O^{(k=1-3)}(12)=1,\left(\frac{2}{3}+\frac{1}{6} S_{12}\right),\left(\frac{1}{3}-\frac{1}{6} S_{12}\right),
$$

and $S_{12}$ is the tensor operator. After doing some algebra, we find the following equation for the two-body energy:

$$
\begin{aligned}
E_{2}= & \frac{2}{\pi^{4} \rho}\left(\frac{h^{2}}{2 m}\right) \sum_{J L S S_{z}} \frac{(2 J+1)}{2(2 S+1)}\left[1-(-1)^{L+S+1}\right]\left|\left\langle\frac{1}{2} \sigma_{z 1} \frac{1}{2} \sigma_{z 2} \mid S S_{z}\right\rangle\right|^{2} \int d r\left\{\left[f_{\alpha}^{(1)^{\prime 2}} a_{\alpha}^{(1)^{2}}\left(k_{f} r\right)\right.\right. \\
& +\frac{2 m}{h^{2}}\left(\left\{V_{c}-3 V_{\sigma}+V_{\tau}-3 V_{\sigma \tau}+2\left(V_{T}-3 V_{\sigma \tau}\right)+2 V_{\tau z}\right\} a_{\alpha}^{(1)^{2}}\left(k_{f} r\right)\right. \\
& \left.\left.+\left[V_{l 2}-3 V_{l 2 \sigma}+V_{l 2 \tau}-3 V_{l 2 \sigma \tau}\right] c_{\alpha}^{(1)^{2}}\left(k_{f} r\right)\right)\left(f_{\alpha}^{(1)}\right)^{2}\right]+\sum_{k=2,3}\left[f_{\alpha}^{(k)^{\prime 2}} a_{\alpha}^{(k)^{2}}\left(k_{f} r\right)\right. \\
& +\frac{2 m}{h^{2}}\left(\left\{V_{c}+V_{\sigma}+V_{\tau}+V_{\sigma \tau}+(-6 k+14)\left(V_{t z}+V_{t}\right)-(k-1)\left(V_{l s \tau}+V_{l s}\right)\right.\right. \\
& \left.+\left[V_{T}+V_{\sigma \tau}+(-6 k+14) V_{t T}\right]\left[2+2 V_{\tau z}\right]\right\} a_{\alpha}^{(k)}{ }^{2}\left(k_{f} r\right) \\
& \left.\left.+\left[V_{l 2}+V_{l 2 \sigma}+V_{l 2 \tau}+V_{l 2 \sigma \tau}\right] c_{\alpha}^{(k)^{2}}\left(k_{f} r\right)+\left[V_{l s 2}+V_{l s 2 \tau}\right] d_{\alpha}^{(k)^{2}}\left(k_{f} r\right)\right) f_{\alpha}^{(k)^{2}}\right] \\
& +\frac{2 m}{h^{2}}\left\{V_{l s}+V_{l s \tau}-2\left(V_{l 2}+V_{l 2 \sigma}+V_{l 2 \sigma \tau}+V_{l 2 \tau}\right)-3\left(V_{l s 2}+V_{l s 2 \tau}\right)\right\} b_{\alpha}^{2}\left(k_{f} r\right) f_{\alpha}^{(2)} f_{\alpha}^{(3)} \\
& \left.+\frac{1}{r^{2}}\left(f_{\alpha}^{(2)}-f_{\alpha}^{(3)}\right)^{2} b_{\alpha}^{2}\left(k_{f} r\right)\right\}
\end{aligned}
$$

where $\alpha=\left\{J, L, S, S_{z}\right\}$ and the coefficient $a_{\alpha}^{(1)^{2}}$, etc., are defined as

$$
\begin{gathered}
a_{\alpha}^{(1)^{2}}(r, \rho, T)=r^{2} I_{L, S_{z}}(r, \rho, T), \\
a_{\alpha}^{(2)^{2}}(r, \rho, T)=r^{2}\left[\beta I_{J-1, S_{z}}(r, \rho, T)+\gamma I_{J+1, S_{z}}(r, \rho, T)\right], \\
a_{\alpha}^{(3)^{2}}(r, \rho, T)=r^{2}\left[\gamma I_{J-1, S_{z}}(r, \rho, T)+\beta I_{J+1, S_{z}}(r, \rho, T)\right],
\end{gathered}
$$




$$
\begin{gathered}
b_{\alpha}^{(2)}(r, \rho, T)=r^{2}\left[\beta_{23} I_{J-1, S_{z}}(r, \rho, T)-\beta_{23} I_{J+1, S_{z}}(r, \rho, T)\right], \\
c_{\alpha}^{(1)^{2}}(r, \rho, T)=r^{2} \nu_{1} I_{L, S_{z}}(r, \rho, T), \\
c_{\alpha}^{(2)^{2}}(r, \rho, T)=r^{2}\left[\eta_{2} I_{J-1, S_{z}}(r, \rho, T)+\nu_{2} I_{J+1, S_{z}}(r, \rho, T)\right], \\
c_{\alpha}^{(3)^{2}}(r, \rho, T)=r^{2}\left[\eta_{3} I_{J-1, S_{z}}(r, \rho, T)+\nu_{3} I_{J+1, S_{z}}(r, \rho, T)\right], \\
d_{\alpha}^{(2)^{2}}(r, \rho, T)=r^{2}\left[\xi_{2} I_{J-1, S_{z}}(r, \rho, T)+\lambda_{2} I_{J+1, S_{z}}(r, \rho, T)\right], \\
d_{\alpha}^{(3)^{2}}(r, \rho, T)=r^{2}\left[\xi_{3} I_{J-1, S_{z}}(r, \rho, T)+\lambda_{3} I_{J+1, S_{z}}(r, \rho, T)\right],
\end{gathered}
$$

with

$$
\begin{gathered}
\beta=\frac{J+1}{2 J+1}, \gamma=\frac{J}{2 J+1}, \beta_{23}=\frac{2 J(J+1)}{2 J+1}, \\
\nu_{1}=L(L+1), \nu_{2}=\frac{J^{2}(J+1)}{2 J+1}, \nu_{3}=\frac{J^{3}+2 J^{2}+3 J+2}{2 J+1}, \\
\eta_{2}=\frac{J\left(J^{2}+2 J+1\right)}{2 J+1}, \eta_{3}=\frac{J\left(J^{2}+J+2\right)}{2 J+1}, \\
\xi_{2}=\frac{J^{3}+2 J^{2}+2 J+1}{2 J+1}, \xi_{3}=\frac{J\left(J^{2}+J+4\right)}{2 J+1}, \\
\lambda_{2}=\frac{J\left(J^{2}+J+1\right)}{2 J+1}, \lambda_{3}=\frac{J^{3}+2 J^{2}+5 J+4}{2 J+1},
\end{gathered}
$$

and

$$
I_{J, S_{z}}(r, \rho, T)=\frac{1}{2 \pi^{6} \rho^{2}} \int d k_{1} d k_{2} n_{i}\left(k_{1}, T, \rho_{i}\right) n_{j}\left(k_{2}, T, \rho_{j}\right) J_{J}^{2}\left(\left|k_{2}-k_{1}\right| r\right)
$$

In the above equation $J_{J}(x)$ is the Bessel's function .

Now, we minimize the two-body energy Eq.(13), with respect to the variations in the functions $f_{\alpha}{ }^{(i)}$, but subject to the normalization constraint [45],

$$
\frac{1}{A} \sum_{i j}\left\langle i j\left|h_{S_{z}}^{2}-f^{2}(12)\right| i j\right\rangle_{a}=0 .
$$


In the case of spin polarized neutron matter, the function $h_{S_{z}}(r)$ is defined as

$$
\begin{array}{rlrl}
h_{S_{z}}(r) & =\left[1-\left(\frac{\gamma_{i}(r)}{\rho}\right)^{2}\right]^{-1 / 2} ; S_{z}= \pm 1 \\
& =1 & ; S_{z}=0
\end{array}
$$

where

$$
\gamma_{i}(r)=\frac{1}{2 \pi^{2}} \int n_{i}\left(k, T, \rho_{i}\right) J_{0}(k r) k^{2} d k
$$

From the minimization of the two-body cluster energy, we get a set of coupled and uncoupled differential equations which are the same as presented in Ref. [45], with the coefficients replaced by those indicated in equations (14)-(22). We can obtain correlation functions by solving the differential equations and then the two-body energy is computed.

Finally, we must calculate the total free energy per particle, $F$, to get the macroscopic properties of hot neutron matter,

$$
F=E-T S
$$

where $S$ is the entropy per neutron,

$$
S(\rho, T)=-\frac{1}{N} \sum_{i=1,2} \sum_{k}\left\{\left[1-n_{i}\left(k, T, \rho_{i}\right)\right] \ln \left[1-n_{i}\left(k, T, \rho_{i}\right)\right]+n_{i}\left(k, T, \rho_{i}\right) \ln n_{i}\left(k, T, \rho_{i}\right)\right\}
$$

We introduce the effective masses, $m_{i}^{*}$, as variational parameters [39, 40, 41, 42, 43]. In fact, we minimize the free energy with respect to the variations in the effective masses and then, we obtain the chemical potentials and the effective masses of spin-up and spin-down neutrons at the minimum point of the free energy. This minimization is done numerically.

\section{RESULTS}

In Fig. 1, we have plotted the effective mass of spin-up and spin-down neutrons versus spin polarization $(\delta)$ for fixed density $\rho=0.16 \mathrm{fm}^{-3}$ at $T=10$ and $T=20 \mathrm{MeV}$. We see that the effective masses of the above components have the same values at $\delta=0$ and get different values by increasing the spin polarization. It can be seen that the effective mass

of spin-up neutrons is larger than spin-down neutrons for $\delta>0$ and the effective mass of spin-down (spin-up) neutrons decreases (increases) by increasing the polarization. In Fig. 
1, we have also included the results of BPRRV calculations [31] for comparison. It is seen that the behavior of the effective mass versus $\delta$ obtained from our method shows the same properties as BPRRV calculations [31].

Our results indicate that the effective masses of spin-up and spin-down neutrons increase by increasing the temperature. Whereas, the results of BPRRV [31] and Isayev [46] show that the effective masses of nucleons in the spin polarized neutron matter and nuclear matter are decreasing functions of the temperature. However, in the references [31] and [46], the authors determine the momentum independent effective masses through the derivatives of the self-consistently calculated single particle potentials at the corresponding Fermi momenta while, in our calculations, the effective masses are introduced in the single particle energies and considered as variational parameters for the free energy. We note that increasing the effective mass by increasing the temperature can be also seen in the results of others where the procedures for finding the effective mass are the same as ours [39, 40, 41, 43].

The behavior of the free energy per particle of the polarized hot neutron matter versus total number density $(\rho)$ for different values of the spin polarization $(\delta)$ at $T=10$ and $T=20 \mathrm{MeV}$ is shown in Fig. 2. This figure shows that the free energy increases with increasing both density and polarization. It is seen that the minimum value of free energy occurs for unpolarized case $(\delta=0.0)$ at any density and at relevant considered temperature. By comparison, in the two panels of Fig. 2, we see that the free energy decreases by increasing temperature. It is also seen that there is no crossing of the free energy curves for different polarizations. Conversely, by increasing the density, the difference between the free energy of neutron matter at different polarizations becomes more appreciable. According to this result, the spontaneous phase transition to a ferromagnetic state in the hot neutron matter does not occur. If such a transition existed, a crossing of the energies of different polarizations would have been observed at some density, indicating that the ground state of the system would be ferromagnetic from that density on.

In Fig. 3, we have presented the free energy per particle as a function of the quadratic spin polarization $\left(\delta^{2}\right)$ for fixed density $\rho=0.36 \mathrm{fm}^{-3}$ at $T=10$ and $T=20 \mathrm{MeV}$. It can be seen that the free energy per particle increases by increasing the polarization. We see that the variation of the free energy of hot neutron matter versus $\delta^{2}$ is nearly linear. This indicates that the ground state of hot neutron matter is paramagnetic. In Fig. 3, we have compared our results with those of BPRRV calculations [31]. It is seen that there is an 
overall agreement between our results and the results of BPRRV [31].

The magnetic susceptibility, $\chi$, which characterizes the response of a system to the magnetic field is given by

$$
\chi=\frac{\mu^{2} \rho}{\left(\frac{\partial^{2} F}{\partial \delta^{2}}\right)_{\delta=0}},
$$

where $\mu$ is the magnetic moment of neutron. In Fig. 4, we have shown the ratio $\chi_{F} / \chi$ as a function of the total number density at two values of temperature $T=10$ and $T=20$ $\mathrm{MeV} . \chi_{F}$ is the magnetic susceptibility for a free Fermi gas. As can be seen from Fig. 4 , even at high densities, the ratio $\chi_{F} / \chi$ increases monotonically and continuously as the density increases for any temperature. This shows that there is no magnetic instability in hot neutron matter. In Fig. 4, the results of BPRRV calculations [31] at $T=20 \mathrm{MeV}$ are also presented for comparison. We see that our results and BPRRV results [31] have an agreement at low densities.

In Fig. 5, the difference of the entropy per particle of fully polarized and unpolarized cases is plotted as a function of the total number density at $T=10$ and $T=20 \mathrm{MeV}$. Fig. 5 shows that for all relevant densities, this difference has negative values. According to this result, we can conclude that the fully polarized case is more ordered than the unpolarized case. In Fig. 5, the results of BPRRV calculations [31] are also given for comparison. There is an agreement between our results and the BPRRV [31] results at low densities.

In Fig. 6, we have plotted the entropy per particle of hot neutron matter versus spin polarization for fixed density $\rho=0.32 \mathrm{fm}^{-3}$ and temperature $T=20 \mathrm{MeV}$. It is seen that the entropy decreases as polarization increases with its highest value occurring for the unpolarized case. To prevent anomalous behavior of entropy as a function of spin polarization, for a given density and temperature, a condition for effective masses can be derived [29],

$$
\frac{m^{*}(\rho, \delta=1.0)}{m^{*}(\rho, \delta=0.0)}<2^{2 / 3}
$$

where $m^{*}(\rho, \delta=1.0)$ and $m^{*}(\rho, \delta=0.0)$ are the effective masses of the fully polarized and unpolarized neutron matter, respectively. From Fig. 1, we have found out that for density $\rho=0.16 \mathrm{fm}^{-3}$, the above ratio at $T=10 \mathrm{MeV}$ is $\frac{m_{1}^{*}(\delta=1.0)}{m_{1,2}^{*}(\delta=0.0)}=1.22$ and at $T=20 \mathrm{MeV}$ is $\frac{m_{1}^{*}(\delta=1.0)}{m_{1,2}^{*}(\delta=0.0)}=1.18$ which are smaller than the indicated limit. This condition is satisfied for 
all other densities explored in this work. Therefor, we can see that the entropy of polarized case is always smaller than the entropy of unpolarized case.

The equation of state of hot polarized neutron matter, $P(\rho, T, \delta)$, can be simply obtained using

$$
P(\rho, T, \delta)=\rho^{2} \frac{\partial F(\rho, T, \delta)}{\partial \rho}
$$

In Fig. 7, we have presented the pressure of neutron matter as a function of the total number density $(\rho)$ for different polarizations at $T=10$ and $T=20 \mathrm{MeV}$. We see that the equation of state becomes stiffer by increasing the polarization.

\section{SUMMARY AND CONCLUSIONS}

Some thermodynamic properties of the polarized neutron matter at finite temperature were reexamined using the lowest order constrained variational (LOCV) method employing the $A V_{18}$ nucleon-nucleon potential. Our main goal was to check the occurrence of the spontaneous transition to the ferromagnetic state. We found no indication for the occurrence the ferromagnetic phase, in agreement with the results of others who used the different manybody techniques. Effective mass, free energy per particle, magnetic susceptibility, entropy per particle, and the equation of state for the polarized neutron matter at finite temperature were calculated and the effect of polarization on these properties were examined.

\section{Acknowledgments}

This work has been supported by Research Institute for Astronomy and Astrophysics of Maragha. We wish to thank Shiraz University and Zanjan University Research Councils. We also wish to thank A. Poostforush for various useful discussion.

[1] S. Shapiro and S. Teukolsky, Blak Holes, White Dwarfs and Neutron Stars, (Wiley-New York, 1983).

[2] F. Pacini, Nature (London) 216 (1967) 567.

[3] T. Gold, Nature (London) 218 (1968) 731. 
[4] J. Navarro, E. S. Hernandez and D. Vautherin, Phys. Rev. C 60 (1999) 045801.

[5] A. Burrows and J. M. Lattimer, Astrophys. J. 307 (1968) 178.

[6] D. H. Brownell and J. Callaway, Nuovo Cimento B 60 (1969) 169.

[7] M. J. Rice, Phys. Lett. A 29 (1969) 637.

[8] J. W. Clark and N. C. Chao, Lettere Nuovo Cimento 2 (1969) 185.

[9] J. W. Clark, Phys. Rev. Lett. 23 (1969) 1463.

[10] S. D. Silverstein, Phys. Rev. Lett. 23 (1969) 139.

[11] E. Østgaard, Nucl. Phys. A 154 (1970) 202.

[12] J. M. Pearson and G. Saunier, Phys. Rev. Lett. 24 (1970) 325.

[13] V. R. Pandharipande, V. K. Garde and J. K. Srivastava, Phys. Lett. B 38 (1972) 485.

[14] S. O. Backman and C. G. Kallman, Phys. Lett. B 43 (1973) 263.

[15] P. Haensel, Phys. Rev. C 11 (1975) 1822.

[16] A. D. Jackson, E. Krotscheck, D. E. Meltzer and R. A. Smith, Nucl. Phys. A 386 (1982) 125.

[17] M. Kutschera and W. Wojcik, Phys. Lett. B 223 (1989) 11.

[18] S. Marcos, R. Niembro, M. L. Quelle and J. Navarro, Phys. Lett. B 271 (1991) 277.

[19] P. Bernardos, S. Marcos, R. Niembro and M. L. Quelle, Phys. Lett. B 356 (1995) 175.

[20] A. Vidaurre, J. Navarro and J. Bernabeu, Astron. Astrophys. 35 (1984) 361.

[21] M. Kutschera and W. Wojcik, Phys. Lett. B 325 (1994) 271.

[22] S. Fantoni, A. Sarsa and K. E. Schmidt, Phys. Rev. Lett. 87 (2001) 181101.

[23] I. Vidana, A. Polls and A. Ramos, Phys. Rev. C 65 (2002) 035804.

[24] I. Vidana and I. Bombaci, Phys. Rev. C 66 (2002) 045801.

[25] W. Zuo, U. Lombardo and C.W. Shen, in Quark-Gluon Plasma and Heavy Ion Collisions, Ed. W.M. Alberico, M. Nardi and M.P. Lombardo, World Scientific, p. 192 (2002).

[26] A. A. Isayev and J. Yang, Phys. Rev. C 69 (2004) 025801.

[27] W. Zuo, U. Lombardo and C. W. Shen, nucl-th/0204056.

W. Zuo, C. W. Shen and U. Lombardo, Phys. Rev. C 67 (2003) 037301.

[28] A. Akmal, V. R. Pandharipande and D. G. Ravenhall, Phys. Rev. C 58 (1998) 1804.

[29] A. Rios, A. Polls and I. Vidana, Phys. Rev. C 71(2005) 055802.

[30] D. Lopez-Val, A.Rios, A. Polls and I. Vidana, Phys. Rev. C 74 (2006) 068801.

[31] I. Bombaci, A. Polls, A. Ramos, A. Rios and I. Vidana, Phys. Lett. B 632 (2006) 638.

[32] G. H. Bordbar and M. Bigdeli, Phys. Rev. C 75 (2007) 045804. 
[33] G. H. Bordbar and M. Bigdeli, Phys. Rev. C 76 (2007) 035803.

[34] G. H. Bordbar and M. Bigdeli, Phys. Rev. C 77 (2008) 015805.

[35] R. B. Wiringa, V. Stoks and R. Schiavilla, Phys. Rev. C 75 (1995) 38.

[36] V.G.J. Stoks, R.A.M. Klomp, C.P.F. Terheggen and J.J. de Swart, Phys. Rev.C 49 (1994) 2950.

[37] I.E. Lagaris and V.R. Pandharipande, Nucl. Phys. A 359 (1981) 331.

[38] R.B. Wiringa, R.A. Smith and T.L. Ainsworth, Phys. Rev. C 29 (1984) 1207.

[39] M. Modarres, J. Phys. G: Nucl. Part. Phys. 19 (1993) 1349.

[40] M. Modarres, J. Phys. G: Nucl. Part. Phys. 21 (1995) 351.

[41] M. Modarres, J. Phys. G: Nucl. Part. Phys. 23 (1997) 923.

[42] M. Modarres and G. H. Bordbar, Phys. Rev. C 58 (1998) 2781.

[43] B. Friedman and V.R. Pandharipande, Nucl. Phys. A 361 (1981) 502.

[44] G. H. Bordbar, M. Modarres, J. Phys. G: Nucl. Part. Phys. 23 (1997) 1631.

[45] G. H. Bordbar and M. Modarres, Phys. Rev. C 57 (1998) 714.

[46] A.A. Isayev, Phys. Rev. C 76 (2007) 047305. 


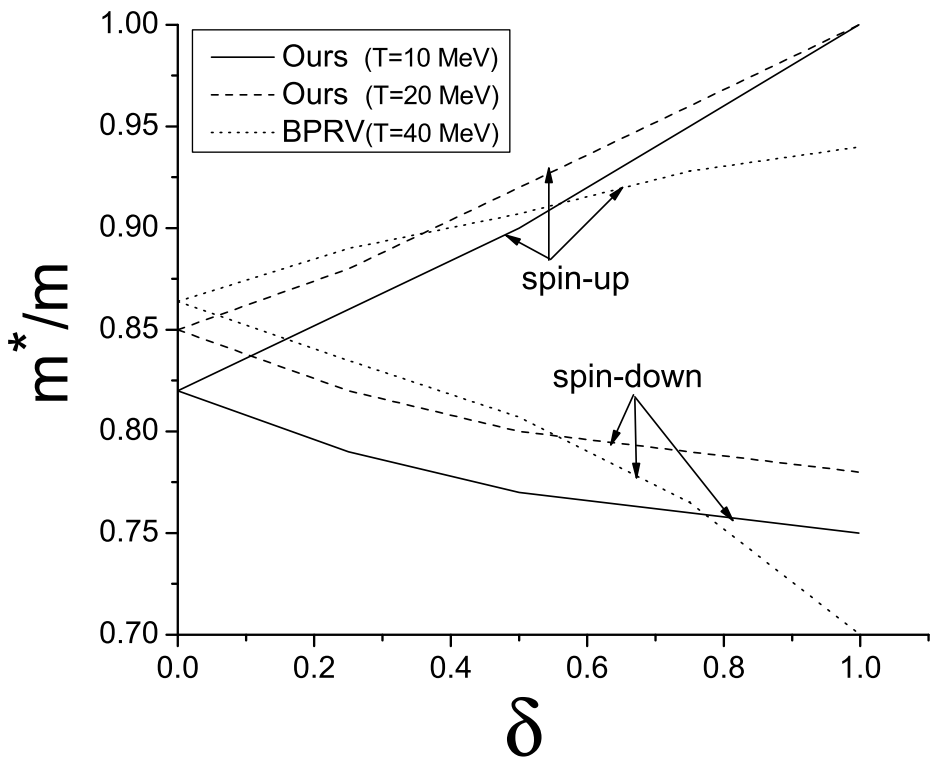

FIG. 1: Our results for the effective mass of spin-up and spin-down neutrons versus spin polarization ( $\delta$ ) for density $\rho=0.16 \mathrm{fm}^{-3}$ at $T=10$ and $T=20 \mathrm{MeV}$. The results of BPRV calculations [31] are also given for comparison. 

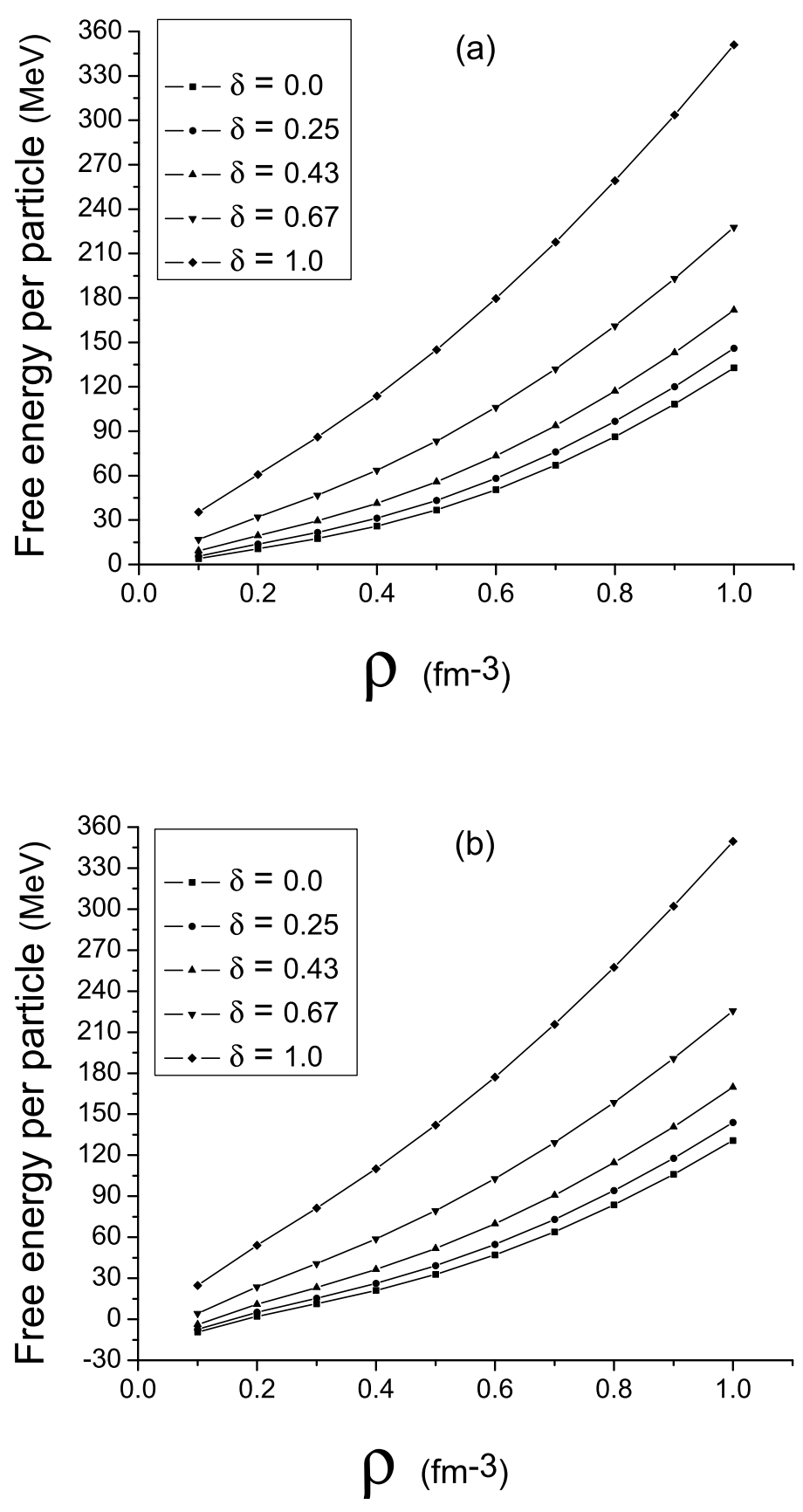

FIG. 2: The free energy per particle of polarized hot neutron matter as a function of the total number density $(\rho)$ for different values of the spin polarization $(\delta)$ at $T=10($ a) and $T=20 \mathrm{MeV}$ (b). 


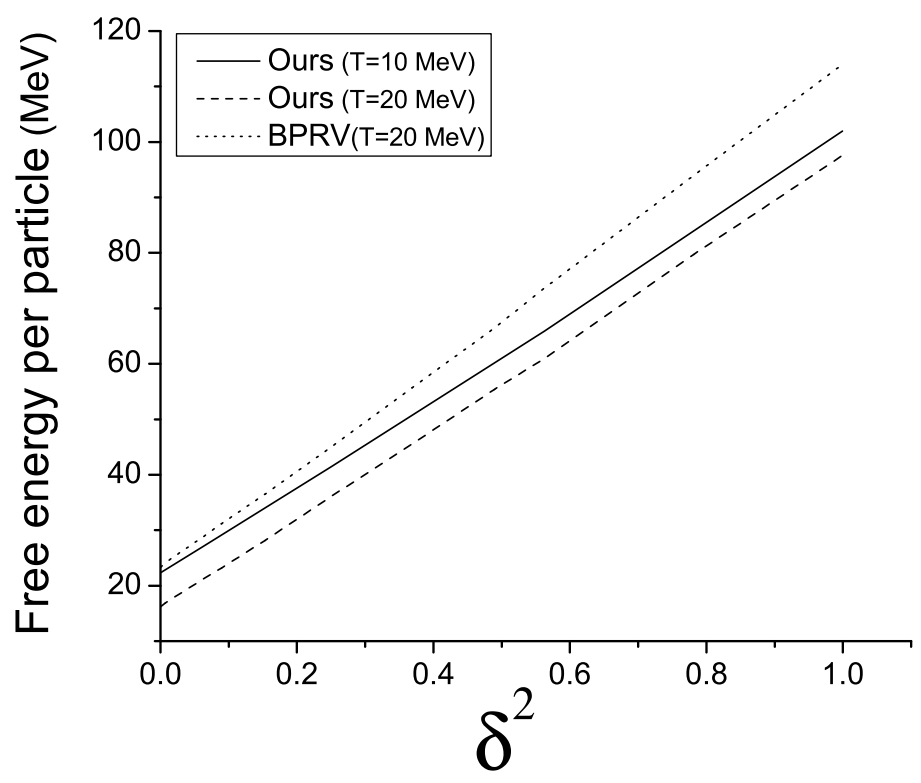

FIG. 3: Our results for the free energy as a function of the quadratic spin polarization $\left(\delta^{2}\right)$ at $T=10 \mathrm{MeV}$ (full curve) and $T=20 \mathrm{MeV}$ (dashed curve) for $\rho=0.36 \mathrm{fm}^{-3}$. The results of BPRV [31] (dashed curve) are also given for comparison. 


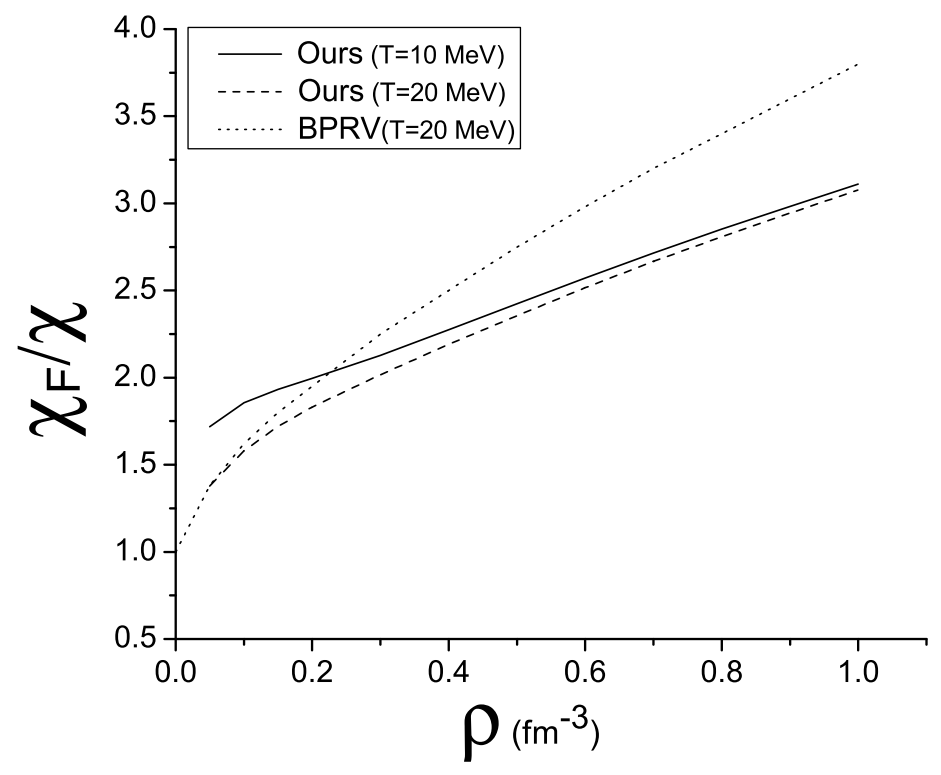

FIG. 4: The magnetic susceptibility of the hot neutron matter versus total number density $(\rho)$ at $T=10 \mathrm{MeV}$ (full curve) and $T=20 \mathrm{MeV}$ (dashed curve). The results of BPRV [31] (dotted curve) are also given for comparison .

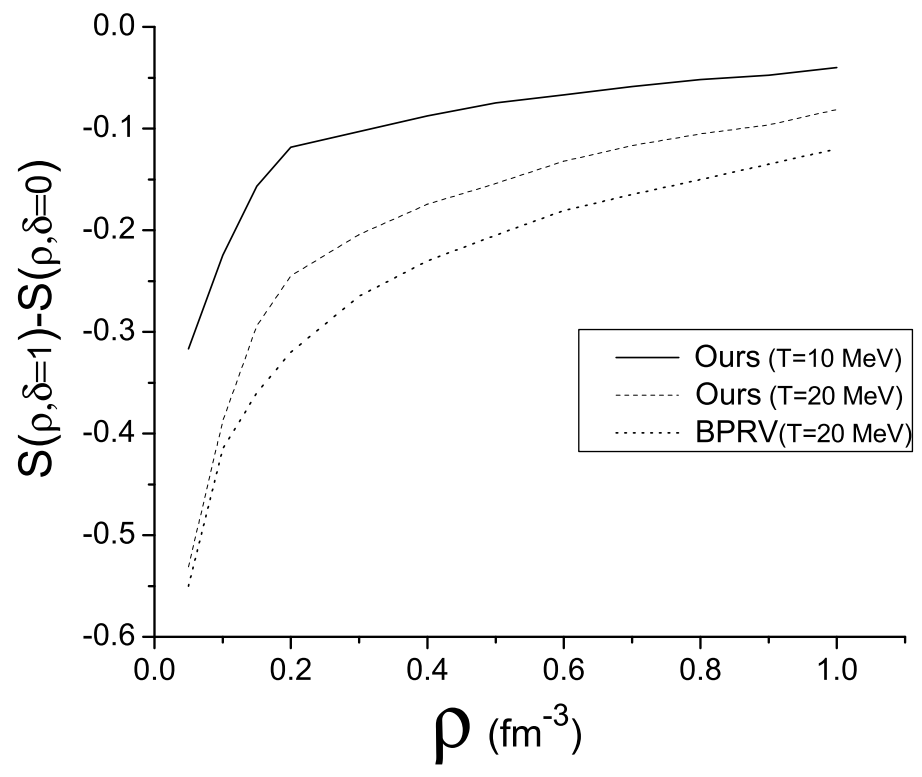

FIG. 5: As Fig. 4 but for the entropy difference of fully polarized and unpolarized cases . 


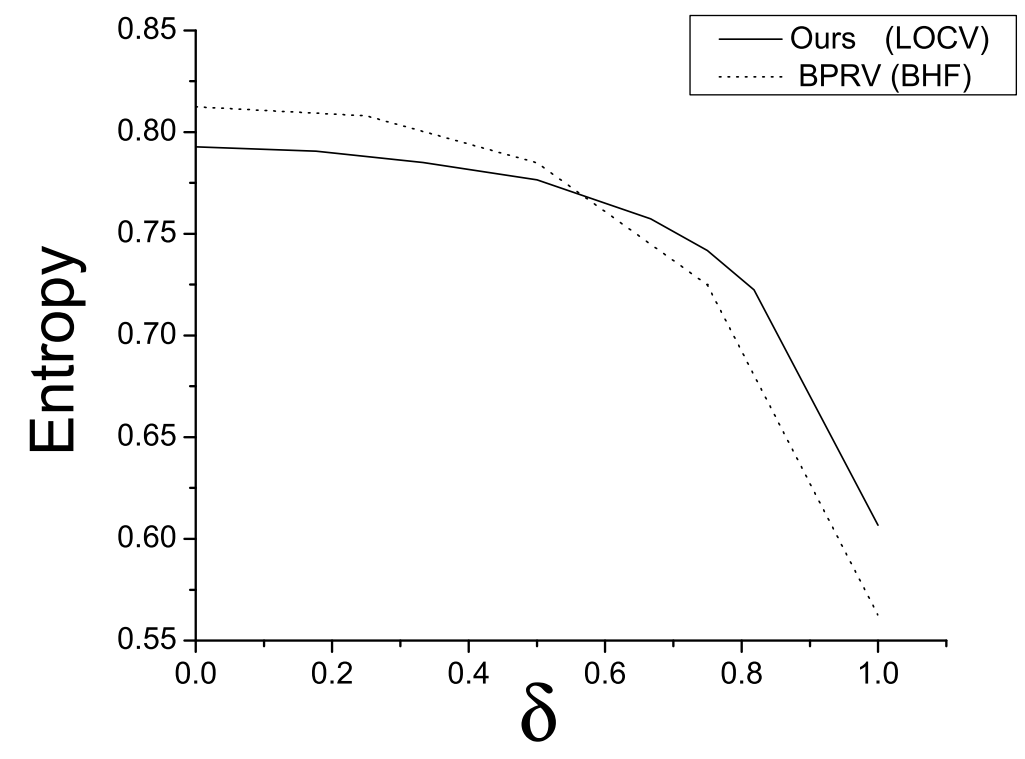

FIG. 6: Our results (full curve) for the entropy per particle as a function of the spin polarization $(\delta)$ at $T=20 \mathrm{MeV}$ and $\rho=0.32 \mathrm{fm}^{-3}$. The results of BPRV [31] (dashed curve) are also given for comparison. 

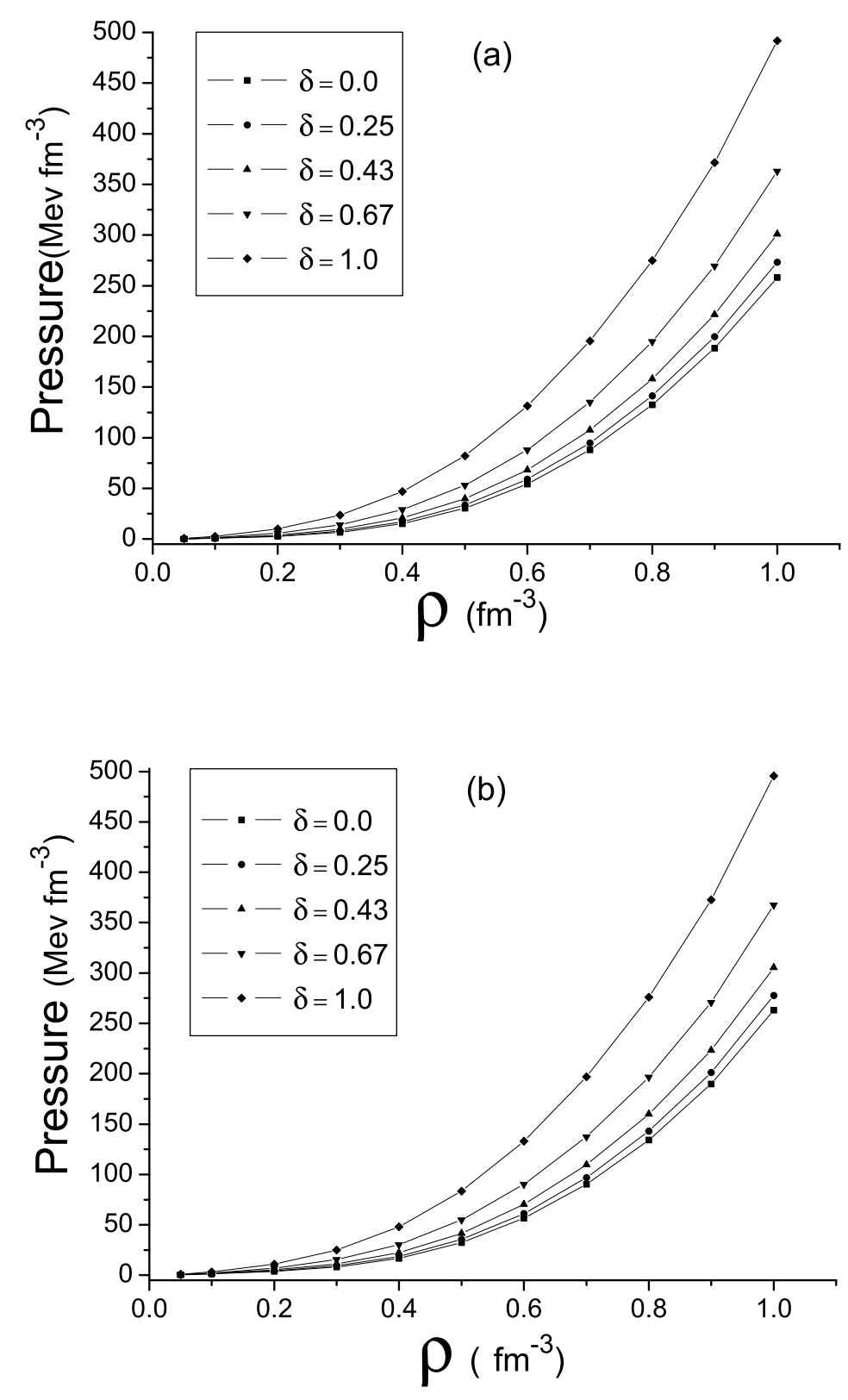

FIG. 7: The equation of state of the hot neutron matter for different values of the spin polarization $(\delta)$ at $T=10$ (a) and $T=20 \mathrm{MeV}(\mathrm{b})$. 